\title{
Acanthamoeba species keratitis in a soft contact lens wearer molecularly linked to well water
}

\author{
Samira Mubareka $M D^{1}$, Michelle Alfa $\mathrm{PhD}^{1}$, Godfrey K Harding $\mathrm{MD}^{1}$, \\ Gregory Booton $\mathrm{PhD}^{2}$, Marilyn Ekins $\mathrm{MD}^{3}$, Paul VanCaeseele $\mathrm{MD}^{1}$
}

\begin{abstract}
S Mubareka, M Alfa, GK Harding, G Booton, M Ekins, $P$ VanCaeseele. Acanthamoeba species keratitis in a soft contact lens wearer molecularly linked to well water. Can J Infect Dis Med Microbiol 2006;17(2):120-122.

Acanthamoeba species keratitis has been associated with soft contact lens wear. In the present report, an epidemiological link was established between the patient's isolate and well water from the home using molecular methods. To the authors' knowledge, this is the first case in Canada where such a link has been established. Primary care practitioners and specialists, including ophthalmologists and infectious diseases specialists, must maintain a high degree of clinical suspicion in soft contact lens wearers with keratitis unresponsive to conventional topical and systemic treatment.
\end{abstract}

\section{Kératite à Acanthamoeba moléculairement liée l'eau d'un puits, chez une porteuse de lentilles cornéennes souples}

Key Words: Acanthamoeba species; Keratitis; Soft contact lens

\section{CASE PRESENTATION}

An otherwise-healthy 14-year-old female soft contact lens wearer, who lived in a rural area where the tap water source for the home was a well, presented to a community hospital with a four-week history of progressive redness, swelling and blurred vision of her left eye despite treatment with topical neomycin and corticosteroids. A dendritiform corneal epithelial lesion, corneal edema and inflammation in the anterior chamber were noted on initial examination, and treatment for herpes simplex keratouveitis with oral valacyclovir and topical trifluridine $1 \%$ was initiated. There was no clinical improvement, and six weeks after the onset of symptoms she was referred to a corneal specialist for evaluation and further management. The patient's visual acuity was 20/80 and her corneal sensation was intact; slit-lamp examination revealed diffuse corneal edema, areas of punctate epithelial staining, poorly defined subepithelial and anterior stromal infiltrates, a partial ring infiltrate and $2+$ cell and flare in the anterior chamber (Figure 1). The patient underwent corneal debridement, and scrapings were sent for viral, bacterial and Acanthameoba culture. She was admitted to the hospital, and polyhexamethalene biguanide $0.02 \%$ and diamidine propamidine isothianate eye drops were added to antiviral therapy and topical neomycin. Over the next few days, her visual acuity decreased to hand movement only, the corneal edema intensified, and midstromal peripheral vascularization developed. Her corneal epithelium healed promptly following debridement. Treatment with oral itraconazole, topical chlorhexidine $0.02 \%$ and topical ciprofloxacin were added after Acanthameoba species were isolated from corneal scrapings. Over the next few months, the patient improved markedly (her left visual acuity was 20/50) despite significant corneal vascularization and persisting central stromal opacity.

Clinical samples, including corneal scrapings, contact lenses, contact lens solution, tap water and well water, were inoculated directly onto non-nutrient agar with Escherichia coli overlay (1), as well as sheep's blood agar, MacConkey agar and chocolate agar. The non-nutrient agar with E coli overlay was incubated at room temperature. The agar surface of the plates was examined daily with an inverted microscope at 10x magnification. On day 5 after planting, the plates inoculated by corneal scrapings were positive for both the cyst (Figure 2) and trophozoite forms of Acanthamoeba species. In addition, Serratia marcescens and Stenotrophomonas maltophilia were isolated from corneal scrapings that were inoculated onto sheep's blood agar, MacConkey agar and chocolate agar. Environmental samples consisting of well water and tap water from the patient's home, in addition to samples from the contact lens case, were also positive for Acanthamoeba species. Acanthamoeba species isolates were forwarded to the Ohio State University Department of Molecular Genetics (Columbus, Ohio, USA), where molecular epidemiological analysis was undertaken. This consisted of amplification and sequencing of 700 base pairs of the mitochondrial 16S rRNA gene and the full nuclear 18S rRNA gene. Sequencing of the mitochondrial $16 \mathrm{~S} r R N A$ gene revealed that both clinical and environmental isolates had the T4 genotype.

${ }^{1}$ Department of Medical Microbiology and Infectious Diseases, University of Manitoba, Winnipeg, Manitoba; ${ }^{2}$ Department of Molecular Genetics, Ohio State University, Columbus, Ohio, USA; ${ }^{3}$ Department of Ophthalmology, University of Manitoba, Winnipeg, Manitoba

Correspondence: Dr Samira Mubareka, Department of Medical Microbiology and Infectious Diseases, University of Manitoba, Basic Medical Sciences Building, 543-730 William Avenue, Winnipeg, Manitoba R3E OW3. Telephone 204-789-3308, fax 204-789-3926, e-mail

ummubare@cc.umanitoba.ca

Received and accepted for publication January 20, 2006 


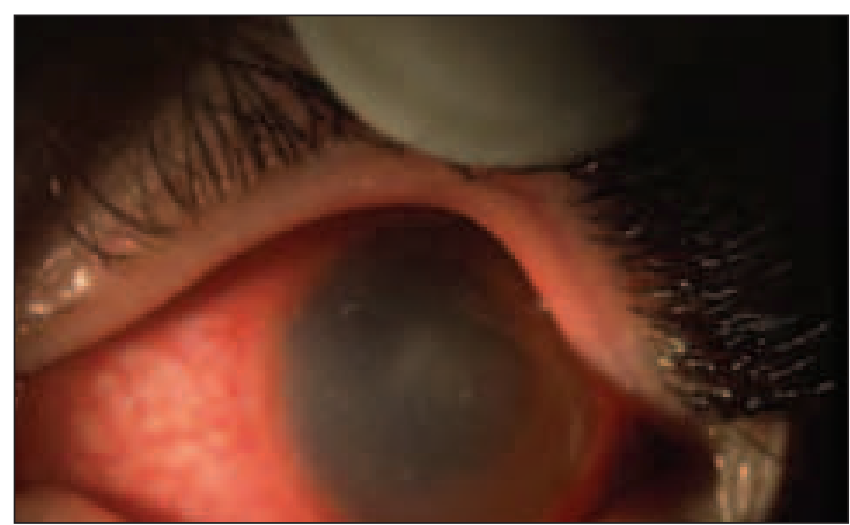

Figure 1) Slit-lamp examination of patient's left eye demonstrating edema and poorly defined infiltrates

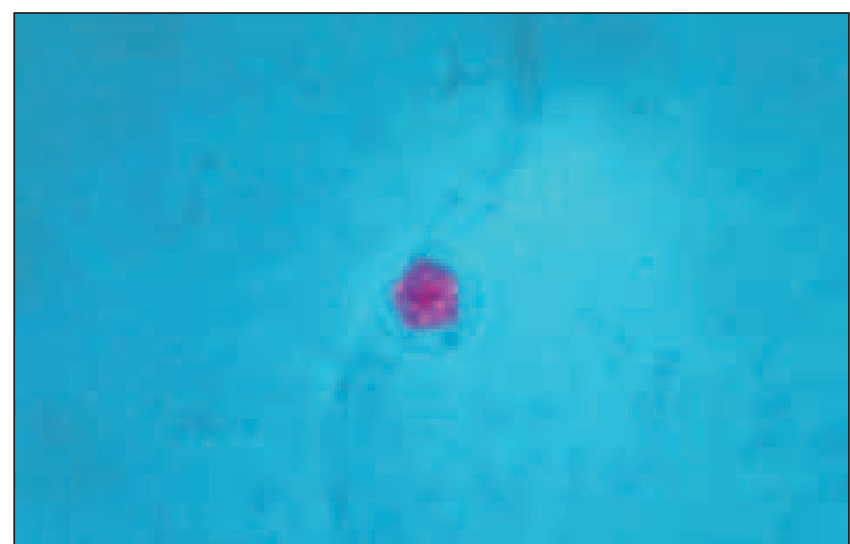

Figure 2) Trichrome stain of Acanthamoeba species cyst seen on non-nutrient agar with Escherichia coli overlay after inoculation of corneal scrapings

In addition, sequencing of the more variable $18 \mathrm{~S} r \mathrm{RNA}$ gene yielded an identical sequence homology for all of the isolates, indicating that both clinical and environmental isolates were the same strain. The phylogenetic gene tree presented is based on an alignment of 2690 sites of the $18 \mathrm{~S} r$ RNA gene (Figure 3). The alignment was performed using the sequence alignment program Eyeball Sequence Editor (ESEE version 3.0, University of Rochester, USA) (2). A total of 2860 sites were used to calculate distances using the Kimura 2-parameter model in the phylogenetic analysis program Molecular Evolutionary Genetics Analysis version 2 (MEGA2, Molecular Evolutionary Genetics Analysis, USA) (3). The tree is rooted with Acanthamoeba species V006, which is a genotype T1 isolate. The isolates from the current study included in the phylogenetic tree are Acanthamoeba species: Canada 03-002, GenBank accession number AY552093 (lens case B); 03-003, GenBank accession number AY552094 (tap water); 03-004, GenBank accession number AY552095 (well water); and 03-005, GenBank accession number AY552096 (left corneal scrape). These isolates are presented in bold-faced type on the gene tree (Figure 3).

Acanthamoeba species are free-living amoebas with a global distribution, and have been isolated from soil as well as a wide variety of water sources, including tap water (4). The Acanthamoeba species' life cycle consists of trophozoite and cyst stages, the latter promoting persistence in both the environment and an infected host. Resistance to chlorination

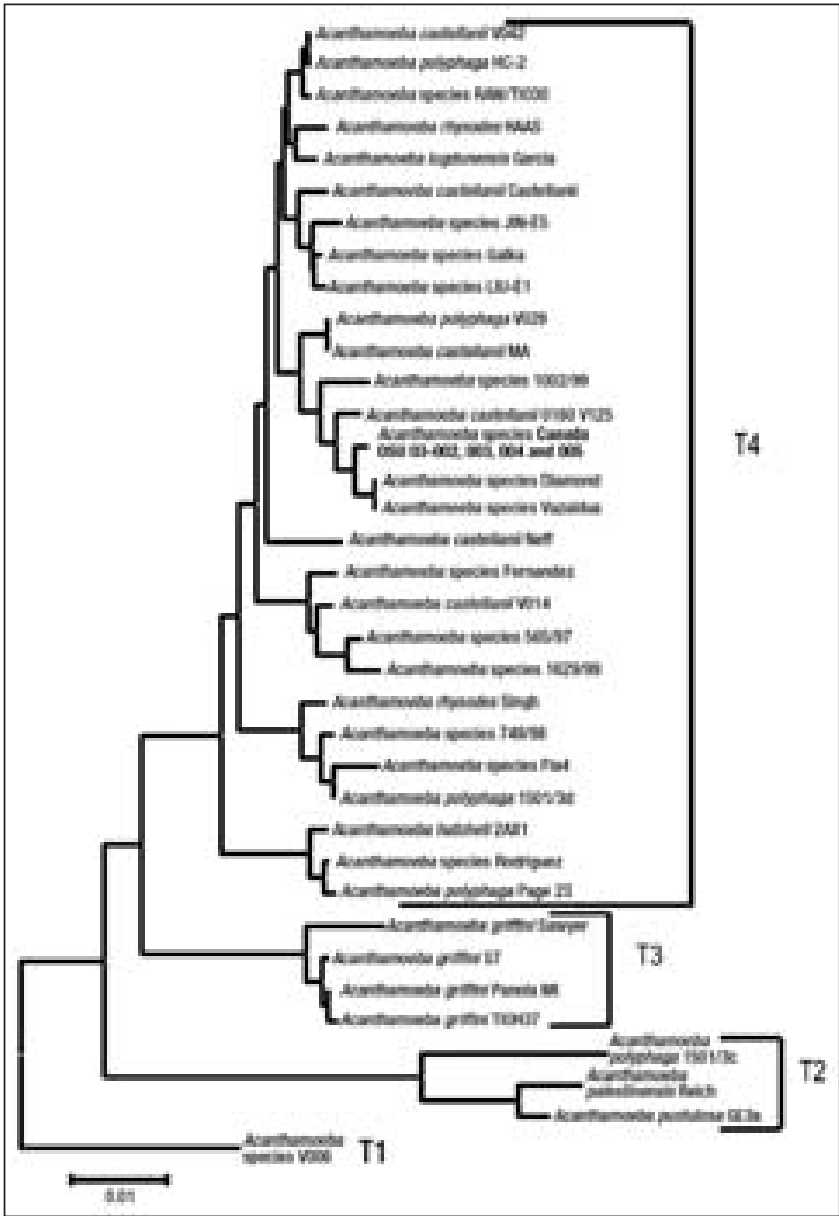

Figure 3) Acanthamoeba species $18 \mathrm{~S}$ rRNA gene tree. The distance scale is the number of base pair substitutions calculated per base pair

permits survival in contact lens paraphernalia after contamination (1). The annual incidence of microbial keratitis associated with daily wear soft contact lenses is estimated to be 1:2500 (5). Risk factors for Acanthamoeba species keratitis (AK) include inappropriate soft contact lens care (6), and exposure to swimming pool water. A British survey (7) spanning two years, studying 106 reported cases of AK, estimated an annual incidence of 17.53 to 21.14 per $1,000,000$ contact lens wearers. Of note, the incidence has been rising since the first descriptions of AK in the 1970s (8). This may be due to a combination of increased soft contact lens wear as well as improved microbiological detection.

Exposure to Acanthamoeba species is common due to its ubiquitous nature. Unlike granulomatous amoebic encephalitis with Acanthamoeba species, subacute and chronic corneal infection is seen in immunocompetent hosts (9). In some cases, the source has been proven to be tap water, which is colonized with Acanthamoeba species, subsequently contaminating contact lenses, which serve as vectors (4). Recent advances in molecular epidemiology of Acanthamoeba species have established clinical and environmental links $(10,11)$. The pathogenesis of $\mathrm{AK}$ is likely secondary to a combination of exposure and trauma. Trauma induced by contact lens wear disrupts the integrity of the corneal epithelium, and preceding trophozoite or oocyst adherence to the contact lens is followed by attachment to the corneal epithelium via mannose-binding proteins, which have been found to be expressed on the surface of 
Acanthamoeba castellanii. Invasion of the underlying stroma via cytolysis and corneal desquamation, and the elaboration of proteases follows at a later stage (12). Acanthapodia-dependent phagocytosis has been demonstrated in pathogenic strains of Acanthamoeba species (13). Radial keratoneuritis is secondary to cytolysis of corneal nerves, and is responsible for the pain generally associated with AK (14). In the current case, the pain was unusually attenuated. This may have been due to early topical corticosteroid application.

Classically, Acanthamoeba species have been identified based on morphological characteristics of cysts and trophozoites (12). Advances in molecular diagnostics have permitted characterization at the genotype level (15) and have established epidemiological links between isolates. Twelve, possibly more, different Acanthamoeba species genotypes have been identified (T1 to T12). One particular genotypic cluster containing T3, T4 and T11 genotypes has been shown to be pathogenic in vitro, demonstrated by growth at higher temperatures, cytotoxicity and elaboration of proteases (11). Genotype T6 has also been associated with AK (16). In the present case, $18 \mathrm{~S} r R N A$ gene sequencing suggests that $\mathrm{AK}$ was secondary to contact lens case colonization with well water containing Acanthamoeba species genotype T4, which has been described in other cases of AK $(10,17)$. Considering the fact that genotype T4 was identified in the present case, the most likely species of Acanthamoeba is A castellanii. Acanthamoeba polyphaga has also been demonstrated to have the same genotype, although this has not been demonstrated consistently and the organism may be considered polyphyletic. By identifying the same genotype in clinical and environmental isolates, we have linked the present case of AK with exposure to contaminated well water from the patient's home.

Topical therapy with cysticidal agents remains the mainstay of therapy $(18,19)$. Polyhexamethylene biguanide has been demonstrated to be effective, especially if applied in conjunction with other agents. Chlorhexidine applied hourly is also

\section{REFERENCES}

1. Schuster F. Cultivation of pathogenic and free-living amebas. Clin Micobiol Rev 2002;15:342-54.

2. Cabot EL, Beckenbach AT. Simultaneous editing of multiple nucleic acid and protein sequences with ESEE. Comput Appl Biosci 1989;5:233-4.

3. Kumar S, Tamura K, Jakobsen I, Nei M. MEGA2: Molecular evolutionary genetics analysis software. Bioinformatics 2001;17:1244-5.

4. Kilvington S, Gray T, Dart J, et al. Acanthamoeba keratitis: The role of domestic tap water contamination in the United Kingdom. Invest Ophthalmol Vis Sci 2004;45:165-9.

5. Liesegang T. Contact lens-related microbial keratitis: Part I: Epidemiology. Cornea 1997;16:125-31.

6. Schaumberg DA, Snow KK, Dana MR. The epidemic of Acanthamoeba keratitis: Where do we stand? Cornea 1998;17:3-10.

7. Radford CF, Minassian DC, Dart JK. Acanthamoeba keratitis in England and Wales: Incidence, outcome, and risk factors. Br J Opthalmol 2002;86:536-42.

8. Wilhelmus KR. Introduction: The increasing importance of Acanthamoeba. Rev Infect Dis 1991;13(Suppl 5):S367-8.

9. Illingworth CD, Cook SD. Acanthamoeba keratitis. Surv Ophthalmol $1998 ; 42: 493-508$

10. Booton GC, Kelly DJ, Chu YW, et al. $18 \mathrm{~S}$ ribosomal DNA typing and tracking of Acanthamoeba species isolates from corneal scrape specimens, contact lenses, lens cases, and home water supplies of Acanthamoeba keratitis patients in Hong Kong. J Clin Microbiol 2002;40:1621-5.

11. Schroeder JM, Booton GC, Hay J, et al. Use of subgenic $18 \mathrm{~S}$ ribosomal DNA PCR and sequencing for genus and genotype effective, and kills both cysts and trophozoites (20). A successful outcome in patients treated medically is noted in up to $88 \%$, the remainder requiring corneal debridement and possibly penetrating keratoplasty. In the latter case, failure has been associated with relapsing Acanthamoeba species infection (21).

Since the present case was described, two more cases of soft contact lens-associated AK have been diagnosed at Misericordia Health Centre, Winnipeg, Manitoba. Acanthamoeba species was isolated from corneal scrapings, and contact lens case washings were inoculated onto non-nutrient agar with E coli overlay. A 16-year-old male patient also had a history of exposure to well water. Well water samples were not available for analysis because the patient had moved to a new residence. In addition, Acanthamoeba species were also isolated from corneal scrapings of a male soft contact lens wearer working on a farm. These cases highlight the importance of appropriate specimen collection and direct plating to an appropriate media.

A high degree of clinical suspicion of $\mathrm{AK}$ is warranted in soft contact lens wearers with keratitis unresponsive to conventional topical and systemic treatment for herpetic, bacterial or fungal keratitis.

To our knowledge, this is the first case of AK to be molecularly linked to Canadian well water. This organism is common in the environment, and molecular typing is helpful in determining the source of the infection and establishing epidemiological links.

ACKNOWLEDGEMENTS: The authors are grateful to the St Boniface General Hospital (Winnipeg, Manitoba) microbiology laboratory staff for their diligence and technical expertise. The work of GB was funded by United States Public Health Service grant EY09073 awarded by the National Eye Institute. This case was presented at the Canadian Infectious Diseases Society Annual Conference, Québec City, Québec, March 7 to 9, 2003.

identification of Acanthamoeba from humans with keratitis and from sewage sludge. J Clin Microbiol 2001;39:1903-11.

12. Visvesvara GS. Classification of Acanthamoeba. Rev Infect Dis 1991;13(Suppl 5):S369-72.

13. Khan NA. Pathogenicity, morphology, and differentiation of Acanthamoeba. Curr Microbiol 2001;43:391-5.

14. Niederkorn JY, Alizadeh H, Leher H, McCulley JP. The pathogenesis of Acanthamoeba keratitis. Microbes Infect 1999;1:437-43.

15. Stothard DR, Schroeder-Diedrich JM, Awwad MH, et al. The evolutionary history of the genus Acanthamoeba and the identification of eight new $18 \mathrm{~S} r$ RNA gene sequence types. J Eukaryot Microbiol 1998;45: 45-54.

16. Walochnik J, Haller-Schober EM, Kolli H, Picher O, Obwaller A, Aspock H. Discrimination between clinically relevant and nonrelevant Acanthamoeba strains isolated from contact lens-wearing keratitis patients in Austria. J Clin Microbiol 2000;38:3932-6.

17. Khan NA, Jarroll EL, Paget TA. Molecular and physiological differentiation between pathogenic and nonpathogenic Acanthamoeba. Curr Microbiol 2002;45:197-202.

18. Lindquist TD. Treatment of Acanthamoeba keratitis. Cornea 1998;17:11-6

19. Hargrave SL, McCulley JP, Husseini Z. Results of a trial of combined propamidine isothianate and neomycin therapy for Acanthamoeba keratitis. Brolene Study Group. Ophthalmology 1999;106:952-7.

20. Kumar R, Lloyd D. Recent advances in the treatment of Acanthamoeba keratitis. Clin Infect Dis 2002;35:434-41.

21. Bacon AS, Frazer DG, Dart JK, Matheson M, Ficker LA, Wright P. A review of 72 consecutive cases of Acanthamoeba keratitis, 1984-1992. Eye 1993;7:719-25. 


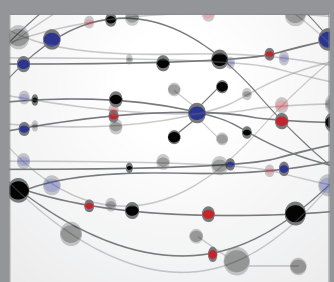

The Scientific World Journal
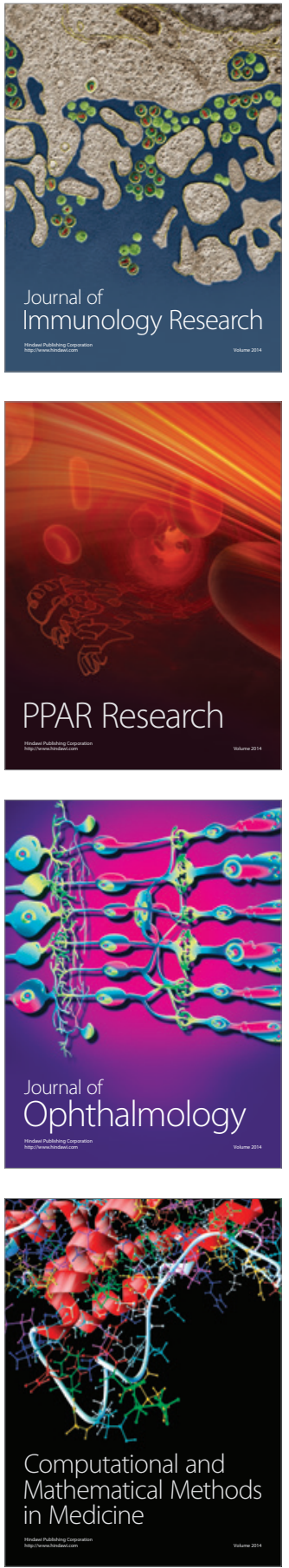

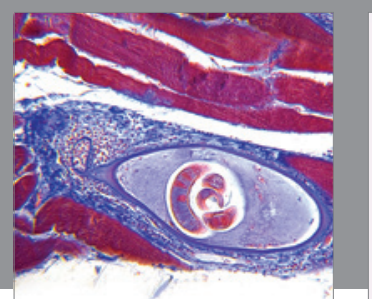

Gastroenterology Research and Practice

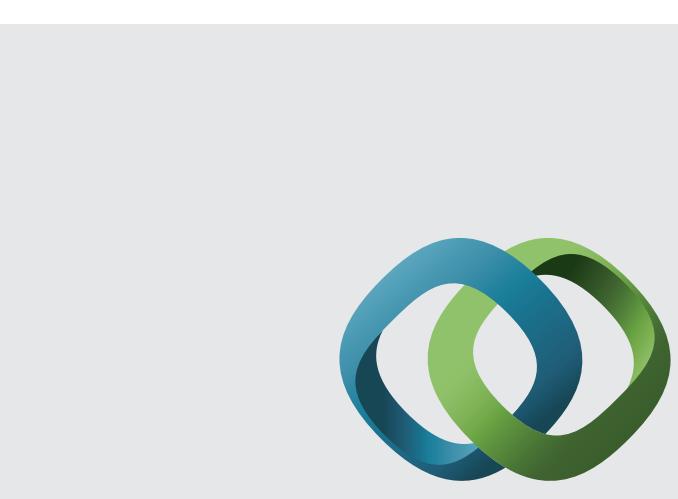

\section{Hindawi}

Submit your manuscripts at

http://www.hindawi.com
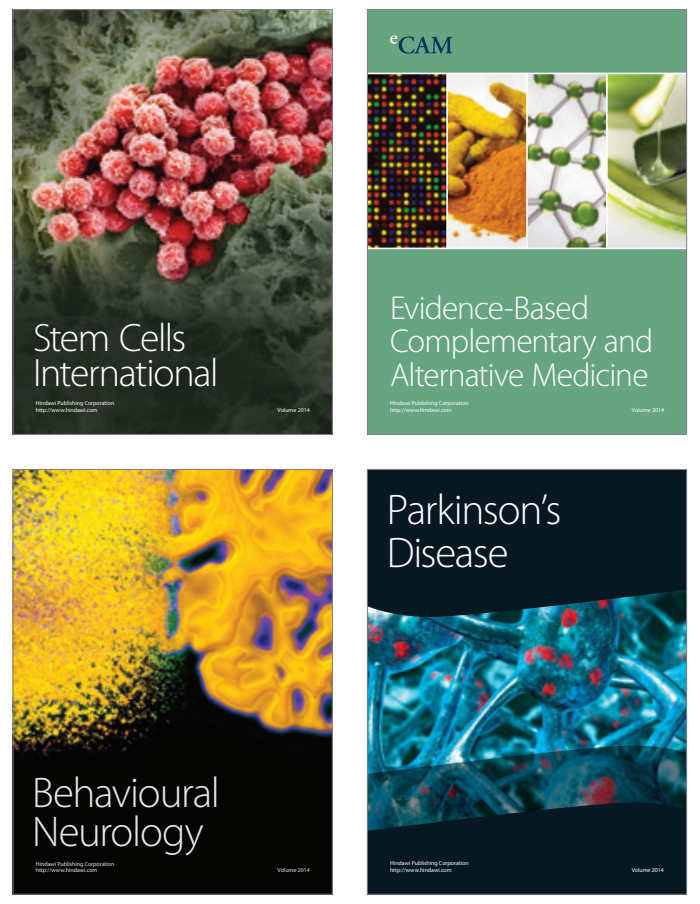
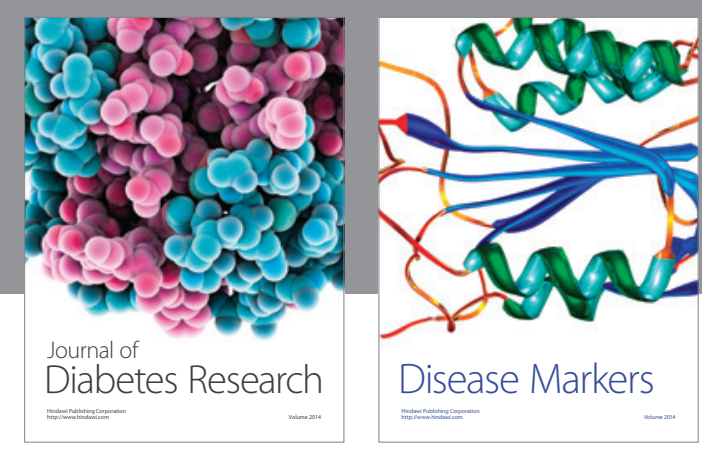

Disease Markers
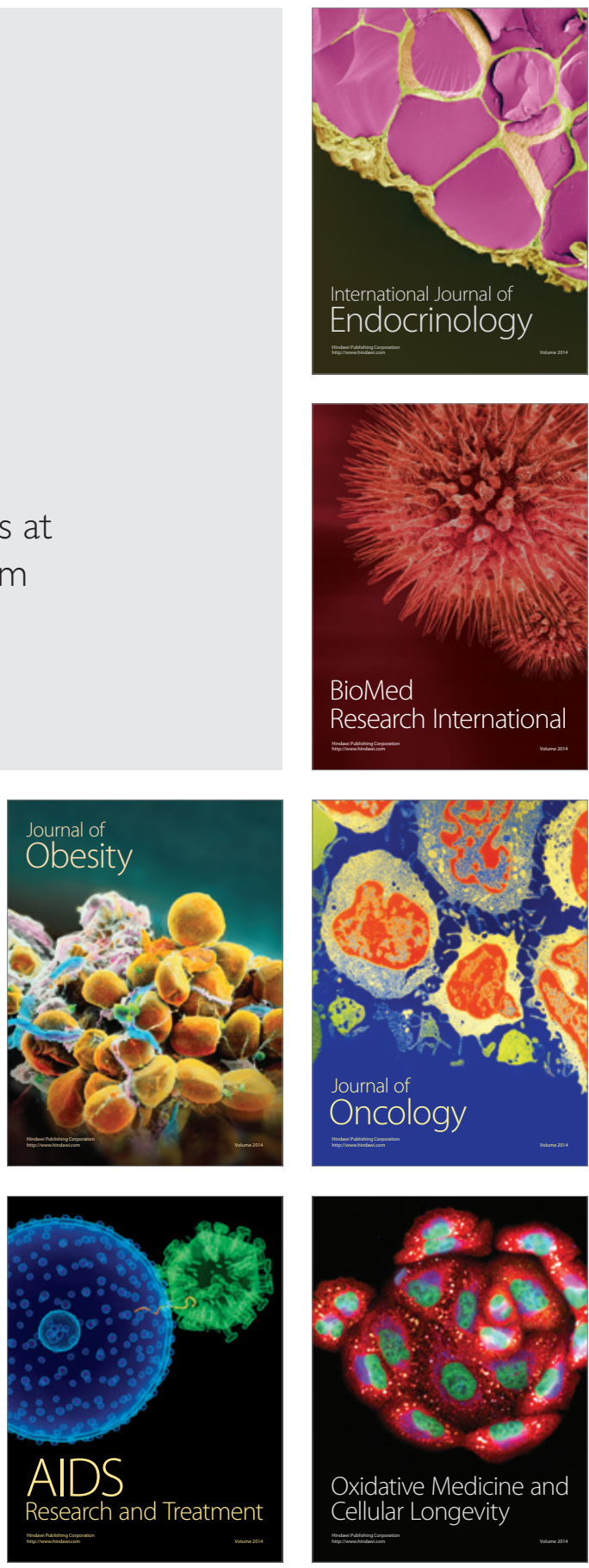